\title{
Development of Stage 4 Macular Hole after Spontaneous Closure in a Patient with Stage 2 Macular Hole and a Lamellar Macular Hole-Associated Epiretinal Proliferation
}

\author{
Masahisa Watanabe Harumasa Yokota Hiroshi Aso \\ Hirotsugu Hanazaki Junya Hanaguri Satoru Yamagami Taiji Nagaoka \\ Division of Ophthalmology, Department of Visual Sciences, Nihon University School of \\ Medicine, Tokyo, Japan
}

\section{Keywords}

Macular hole $\cdot$ Lamellar hole $\cdot$ Epiretinal proliferation $\cdot$ Spontaneous closure

\begin{abstract}
Herein, we report the longitudinal observation of a case with reopening of the macular hole associated with a lamellar macular hole-associated epiretinal proliferation (LHEP) followed by spontaneous closure in patients with stage 2 idiopathic macular hole. A 64-year-old woman was referred for the decreased visual acuity (VA) and acute anorthopia in the right eye. Funduscopy and optical coherence tomography (OCT) showed stage 2 full-thickness macular hole without posterior vitreous detachment (PVD) and operculum formation. Her best-corrected visual acuity (BCVA) was 20/32. One month later, the diameter of the macular hole was getting small and VA improved. Six months later, the macular hole was treated spontaneously with the attached hyaloid membrane to the macula by OCT and the BCVA improved to 20/20. Fourteen months after the first visit, the BCVA decreased to 20/50 and the patient was diagnosed with stage 4 macular hole with complete PVD. OCT showed full-thickness macular hole with a LHEP in the right eye. After 25G-gauge vitrectomy with the peeling of internal limiting membrane (ILM) and LHEP, the macular hole was closed and BCVA finally improved to 20/25. Spontaneous macular hole closure without PVD may rarely occur in patients with LHEP. The surgical removal of ILM and LHEP may contribute to the successful macular hole closure after vitrectomy.
\end{abstract}


Watanabe et al.: Stage $4 \mathrm{MH}$ after Spontaneous Closure

\section{Introduction}

Idiopathic full-thickness macular holes (IMHs) are most commonly caused by anteroposterior vitreofoveal traction. There were some cases to be reported that IMH can spontaneously close without any intervention. Although the mechanism of the spontaneous closure of IMHs have not been fully elucidated, it is widely recognized that the spontaneous release of the vitreomacular traction may account for the closure of IMHs [1,2]. Herein, we report a case of stage 2 macular hole followed by spontaneous closure and reopening of stage 4 macular hole and successfully macular hole closure after vitrectomy.

\section{Case Presentation}

A 64-year-old female was referred for the decreased visual acuity (VA) and acute anorthopia in the right eye. Funduscopy and optical coherence tomography (OCT) showed a stage 2 full-thickness macular hole (minimum diameter $100 \mu \mathrm{m}$ ) without posterior vitreous detachment and operculum formation (Fig. 1a). Her best-corrected visual acuity (BCVA) was 20/32. One month later, the diameter of the macular hole was getting small (Fig. 1b) and VA improved. Six month later, OCT showed that the macular hole was resolved spontaneously with the attached hyaloid membrane to the macula and the hole was sealed with some glialike tissue filling the gap (Fig. 1c). Her BCVA improved to 20/20.

Fourteen months after the first visit, the BCVA decreased to 20/50 and diagnosed with stage 4 macular hole (minimum diameter $117 \mu \mathrm{m}$ ) with complete posterior vitreous detachment. OCT showed that full-thickness macular hole with a lamellar macular hole-associated epiretinal proliferation (LHEP) in the right eye (Fig. 2a). We underwent a 25-gauge pars plana

Fig. 1. Spontaneous closure of stage 2 fullthickness macular hole. Serial OCT images of a 64-year-old female showing spontaneous closure of an untreated stage 2 FTMH at the first visit (a), 1 month later (b), and 6 months later (c). The LHEP was observed at 1 and 6 months later. The size of macular hole was decreased during the follow-up period and spontaneously closed at 6 months after the first visit. OCT, optical coherence tomography; LHEP, lamellar macular hole-associated epiretinal proliferation.

Fig. 2. Reopening and closure of stage 4 macular hole after vitrectomy. Fundus photograph and OCT imaging of the patient's right eye before (a) and 4 weeks after (b) vitrectomy. The LHEP (arrow) was visible around the hole. OCT, optical coherence tomography; LHEP, lamellar macular hole-associated epiretinal proliferation.
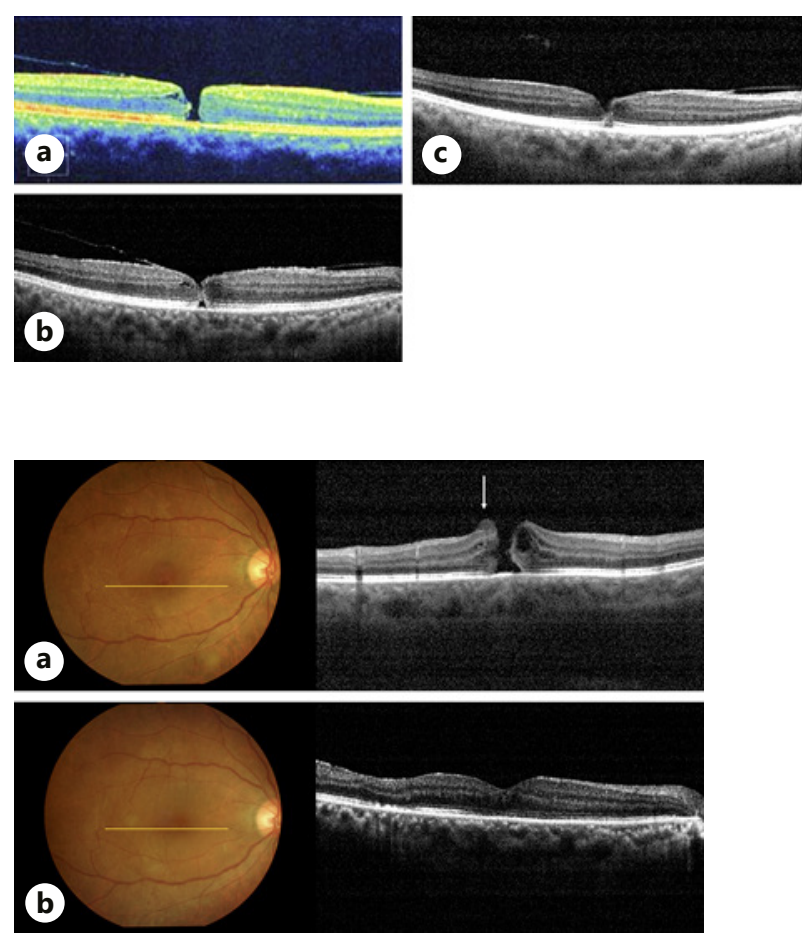
vitrectomy combined with phacoemulsification and intraocular lens implantation, the peeling of both the Brilliant Blue G-assisted internal limiting membrane and LHEP, fluid air exchange, and SF6 gas injection. She was asked to strictly keep face-down position for 7 days after surgery. Postoperatively, OCT showed that macular hole was closed and BCVA improved to 20/40 4 weeks after the surgery (Fig. 2b). At the last visit 30 months after the surgery, her BCVA in the left eye improved to $20 / 25$.

\section{Discussion}

There were some cases to be reported that IMH can spontaneously close without any intervention. It has been reported that the incidence of spontaneous closure of IMH varies from 4 to $11.5 \%$ [3-5]. Hikichi et al. [6] reported that $94 \%$ of eyes with stage 2 IMH were developed to stage 3 or 4 IMH with $>24$ months observation. They also reported that no eye with stage $2 \mathrm{MH}$ had spontaneous closure. Although the mechanism of the spontaneous closure of IMHs have not been fully elucidated, it is widely recognized that the spontaneous release of the vitreoumacular traction may account for the closure of IMHs [1,2]. Petropoulos et al. [7] found epiretinal membrane (ERM) formation during spontaneous closure of IMH. They speculate that the contractile ERM may contribute to the spontaneous closure. In addition, Pang et al. [8] reported that LHEP was found in 8 of 99 eyes (8.0\%) with full-thickness macular hole. They also described that LHEP appeared as a substantial material of homogenous medium reflectivity on the epiretinal surface that demonstrated contiguity with the middle retinal layers and conformed to the adjacent retinal anatomy. Therefore, they postulated that LHEP is primarily driven by a proliferation of Muller cells onto the inner retina that may originate from the middle layers of the retina. In our case, OCT findings clearly showed that a MH gap was filled with a glial-tissue substance which was associated with LHEP. Our case indicated that the LHEP may contribute to the spontaneous macular hole closure because the LHEP already existed when the spontaneous closure occurred (Fig. 1b). Therefore, we speculate that LHEP is the force attempting to seal the hole, while the more widespread ERM is the force to create the reopening of macular hole in our case. Surgical outcome in eyes with a lamellar macular hole and LHEP remains controversial [9]. It is worth noting that LHEP may be associated with the spontaneous closure and reopening of full-thickness macular hole. Although it remains controversial if the LHEP should be completely removed or embedded into the cleavage during vitrectomy, we believe that it should be removed because the LHEP may be associated with poor visual prognosis [10].

\section{Conclusion}

The surgical removal of internal limiting membrane and LHEP may be needed in cases where the eye had recurrent macular hole with LHEP.

\section{Statement of Ethics}

Written informed consent was obtained from the patient for publication of this case report and any accompanying images.

\section{Karger'}


Watanabe et al.: Stage $4 \mathrm{MH}$ after Spontaneous Closure

\section{Conflict of Interest Statement}

The authors have no conflicts of interest to declare.

\section{Funding Sources}

Supported by a Grant-in-Aid for Scientific Research (C) 26861430 from the Ministry of Education, Science, and Culture, Tokyo, Japan (to T.N.).

\section{Author Contributions}

Study design: T.N. and M.W.; performed surgery: T.N., H.Y., H.A., and H.H.; interpretation of the results: S.Y. and J.H.; and drafting of the work: T.N.

\section{References}

1 Imai M, Ohshiro T, Gotoh T, Imasawa M, Iijima H. Spontaneous closure of stage 2 macular hole observed with optical coherence tomography. Am J Ophthalmol. 2003;136(1):187-8.

2 Privat E, Tadayoni R, Gaucher D, Haouchine B, Massin P, Gaudric A. Residual defect in the foveal photoreceptor layer detected by optical coherence tomography in eyes with spontaneously closed macular holes. Am J Ophthalmol. 2007;143(5):814-9.

3 Yuzawa M, Watanabe A, Takahashi Y, Matsui M. Observation of idiopathic full-thickness macular holes. Followup observation. Arch Ophthalmol. 1994;112(8):1051-6.

4 Freeman WR, Azen SP, Kim JW, el-Haig W, Mishell DR 3rd, Bailey I. Vitrectomy for the treatment of fullthickness stage 3 or 4 macular holes. Results of a multicentered randomized clinical trial. The Vitrectomy for Treatment of Macular Hole Study Group. Arch Ophthalmol. 1997;115(1):11-21.

5 Ezra E, Gregor ZJ. Morfields Macular Hole Study Ggroup Report N. Surgery for idiopathic full-thickness macular hole: two-year results of a randomized clinical trial comparing natural history, vitrectomy, and vitrectomy plus autologous serum: Morfields Macular Hole Study Group RAeport no. 1. Arch Ophthalmol. 2004;122(2): 224-36.

6 Hikichi T, Yoshida A, Akiba J, Konno S, Trempe CL. Prognosis of stage 2 macular holes. Am J Ophthalmol. 1995; 119(5):571-5.

7 Petropoulos IK, Matter MA, Desmangles PM. Spontaneous closure of macular hole: one-year follow-up with spectral-domain optical coherence tomography. Klin Monbl Augenheilkd. 2009;226(4):363-4.

8 Pang CE, Spaide RF, Freund KB. Epiretinal proliferation seen in association with lamellar macular holes: a distinct clinical entity. Retina. 2014;34(8):1513-23.

9 Shiode Y, Morizane Y, Takahashi K, Kimura S, Hosokawa M, Hirano M, et al. Embedding of lamellar hole-associated epiretinal proliferation combined with internal limiting membrane inversion for the treatment of lamellar macular hole: a case report. BMC Ophthalmol. 2018;18(1):257.

10 Ubukata Y, Imai H, Otsuka K, Nishizaki M, Hara R, Uenishi M, et al. The comparison of the surgical outcome for the full-thickness macular hole with/without lamellar hole-associated epiretinal proliferation. J Ophthalmol. 2017;2017:9640756. 